\title{
Perfil sociodemográfico e índice de qualidade de vida de cuidadores de pessoas com deficiência física
}

\author{
Socio-demographic profile and quality of life index for caregivers of people with physical disabilities
}

Larissa Coutinho de Lucena Trigueiro, ${ }^{1,}$ eide Maria Gomes de Lucena ${ }^{2}$, Paulo Ortiz Rocha de Aragão ${ }^{3}$, Moema Teixeira Maia Lemos ${ }^{4}$

Estudo desenvolvido no Laboratório de Ergonomia e Saúde do Núcleo de Estudos e Pesquisas Epidemiológicas em Fisioterapia e Saúde do Centro de Ciências da Saúde da Universidade Federal da Paraíba (UFPB) - João Pessoa,(PB), Brasil.

${ }^{1}$ Fisioterapeuta; Mestranda no Programa de Pós-Graduação em Fisioterapia da Universidade Federal do Rio Grande do Norte (UFRN) - Natal (RN), Brasil.

2 Professora Doutora do Curso de Fisioterapia da UFPB - João Pessoa (PB), Brasil.

${ }^{3}$ Professor Doutor do Curso de Pós-Graduação em Sociologia da Universidade Federal de Campina Grande (UFCG) - Campina Grande $(\mathrm{PB})$, Brasil.

${ }^{4}$ Professora; Mestre do Curso de Fisioterapia da UFPB - João Pessoa (PB), Brasil.

ENDERECO PARA CORRESPONDENCIA

Larissa Coutinho de Lucena Trigueiro - Rua Derlópidas Gomes Neves, 115 - Bancários - CEP: 58051-260 - João Pessoa (PB), Brasil - E-mail: larissacoutinho@ gmail.com

APRESENTACÃO ago. 2010

ACEITO PARA PUBLICACÃO mai. 2011

FONTE DE FINANCIAMENTO nenhuma

CONFLITO DE INTERESSES nada a declarar

Parecer de aprovação do Comitê de Ética em Pesquisa do Centro de Ciências da Saúde da UFPB n $600 / 2008$.
RESUMO: O objetivo do estudo foi identificar o perfil sociodemográfico e o nível de qualidade de vida de cuidadores de pessoas com deficiência física atendidas na Fundação Centro Integrado de Apoio ao Portador de Deficiência Física (FUNAD) no município de João Pessoa (PB). Participaram do estudo 51 cuidadores de pessoas com deficiência física, na faixa etária de 19 a 63 anos $(36,29 \pm 11,10)$. Para a avaliação foram utilizados dois instrumentos: um formulário estruturado, com questões sobre o perfil pessoal e social dos cuidadores e o World Health Organization Quality of Life (WHOQOL-bref), para a análise do nível de qualidade de vida. Os resultados apontaram que a maioria dos cuidadores eram mulheres $(92,2 \%)$, mães $(68,6 \%)$, solteiras $(43,1 \%)$ e haviam frequentado a escola até o Ensino Fundamental incompleto $(47 \%)$. Houve significância estatística $(p<0,05)$, com correlação positiva entre idade e tempo de atuação como cuidador atual $(p=0,000)$; e correlação negativa entre escolaridade e tempo de cuidado diário $(p=0,009)$. Constatou-se que o estresse físico e o emocional levam os cuidadores a ficarem sobrecarregados, afetando diretamente sua qualidade de vida. Ressalta-se a importância da investigação do estilo de vida desses cuidadores no intuito de que ações sociais e de saúde sejam desenvolvidas pelas instituições que assistem pessoas com deficiência física.

Descritores: cuidadores; pessoas com deficiência; qualidade de vida.

ABSTRACT: The study objective was to identify the socio-demographic profile and the level of quality of life of caregivers of persons with disabilities met with the Foundation to Support Integrated Patient with Physical Disabilities (FUNAD) in the city of Joao Pessoa, in the state Paraíba, Brazil. The study had 51 participants, caregivers of people with disabilities in the age group 19-63 years $(36.29 \pm 11.10)$. For the evaluation, we used two instruments: a structured form, with questions about personal and social profiles, and World Health Organization Quality of Life (WHOQOL-bref), to analyze the level of quality of life. The results showed that most caregivers were women $(92.2 \%)$, mothers $(68.6 \%)$, single $(43.1 \%)$ and had attended up to elementary school $(47 \%)$. Statistical significance $(p<0.05)$, a positive correlation between age and time working as a caregiver present $(\mathrm{p}=0.000)$ and negative correlation between schooling and daily care time $(p=0.009)$. It was found that the physical and emotional stress leads caregivers to encumber themselves, directly affecting the quality of life. We highlight the importance of research in lifestyle of caregivers, in order that social action and health are developed by institutions assisting people with disabilities.

KeYwords: caregivers; disabled persons; quality of life. 


\section{INTRODUCÃ̃}

Atualmente a deficiência é definida, de acordo com o Decreto $n^{\circ} 3.298 / 99$, como toda perda ou anormalidade de uma estrutura ou função psicológica, fisiológica ou anatômica que gere incapacidade para o desempenho de atividade dentro do padrão considerado normal para o ser humano. Enquadram-se no conceito de deficiência os indivíduos que apresentam uma ou mais dessas ocorrências clínicas: presença de uma anomalia, defeito ou perda de um membro, órgão, tecido ou qualquer estrutura do corpo, inclusive das funções mentais ${ }^{1}$.

Segundo a Organização das Nações Unidas (ONU), o mundo abriga cerca de 500 milhões de pessoas com deficiência, das quais $80 \%$ vivem em países em desenvolvimento. No Brasil, os resultados do Censo de 2000, realizado pelo Instituto Brasileiro de Geografia e Estatística (IBGE), indicam que há aproximadamente 24,6 milhões de brasileiros com algum tipo de incapacidade ou deficiência, o que representa $14,5 \%$ da população total do país. É importante destacar que a proporção de pessoas com deficiência aumenta com a idade, passando de 4,3\% nas crianças até 14 anos para $54 \%$ do total das pessoas com idade superior a 65 anos $^{2,3}$.

Nesse contexto, a família é a unidade básica de suporte a todos aqueles que carecem de cuidados, sejam crianças, adultos ou idosos, com deficiência ou não. A família costuma ser a principal origem do cuidador e as mulheres adultas e idosas preponderam nestes cuidados. Porém, há também registros de cuidadores masculinos e de crianças e adolescentes. Sabe-se, também, que algumas situações costumam determinar esta escolha: proximidade parental (esposas e filhas), física, afetiva e o fato de ser mulher ${ }^{4}$. As tarefas atribuídas ao cuidador, a alteração das rotinas e o tempo despendido no cuidado têm impactos negativos sobre a sua qualidade de vida ${ }^{5}$.

Na história humana, o cuidado está relacionado à sobrevivência, reflete-se na saúde e, consequentemente, na qualidade de vida, sendo definida segundo a Organização Mundial da Saúde (OMS) "como a percepção do indivíduo de sua posição na vida no contexto da cultura e sistema de valores nos quais ele vive e em relação aos seus objetivos, expectativas, padrões e preocupações" ${ }^{\prime 6}$.

Do cuidador é exigido que participe cada vez menos de atividades sociais, seja capaz de resolver problemas e passe por vários momentos de estresse em função dessa rotina de vida. Devido ao nível de envolvimento nos cuidados com o paciente, o cuidador é levado a não prestar atenção nas suas próprias necessidades pessoais, assim, os problemas de natureza emocional e física podem ocorrer manifestando sintomas como tensão, constrangimento, fadiga, estresse, depressão e alteração da autoestima ${ }^{7}$.

O presente estudo teve como objetivo traçar o perfil sociodemográfico e a qualidade de vida de cuidadores de pessoas com deficiência física atendidas na Fundação Centro Integrado de Apoio ao Portador de Deficiência (FUNAD), no município de João Pessoa (PB).

\section{METODOLOGIA}

O estudo é do tipo descritivo, com corte transversal. A população foi composta por 51 cuidadores de pessoas com deficiência física, atendidas na FUNAD, localizada no município de João Pessoa (PB). Os participantes foram selecionados de forma aleatória e de acordo com a acessibilidade de cada um em ser entrevistado e assinar o Termo de Consentimento Livre e Esclarecido (TCLE).

Constituíram-se como critérios de inclusão: ser familiar ou responsável com idade igual ou superior a 18 anos e estar prestando cuidados há mais de 6 meses.

Os critérios de exclusão foram: as famílias nas quais não foi possível identificar o cuidador principal e os indivíduos que não quiseram participar da pesquisa.

Os objetivos do estudo foram explicados e aqueles que concordaram em participar, convidados a se dirigir a uma sala reservada a fim de responder a dois instrumentos e assinarem o TCLE. A coleta dos dados foi realizada no período de dezembro de 2008 a maio de 2009. A técnica utilizada para a coleta de dados foi a de entrevista realizada com o cuidador no momento em que ele aguardava o paciente, que se encontrava em atendimento.
Foram utilizados dois instrumentos nesse estudo. O primeiro formulário, adaptado de Barba et al. ${ }^{7}$, de natureza semiestruturada, contêm 19 questões no total, as quais buscam identificar características sociodemográficas do cuidador: idade, sexo, estado civil, escolaridade, profissão, tempo de atuação como cuidador do paciente, se há ou não grau de parentesco entre o cuidador e o paciente, presença ou não de problemas de saúde com o cuidador, o diagnóstico clínico do paciente, localização de desconforto/presença de sintomatologia dolorosa e a intensidade desse desconforto/dor do cuidador, por meio da Escala de Borg.

O segundo formulário utilizado, a fim de avaliar a qualidade de vida dos cuidadores, foi a versão abreviada do questionário original World Health Organization Quality of Life (WHOQOL100), denominado WHOQOL-bref. O instrumento foi validado e traduzido para a língua portuguesa por Chachamovich et al., os quais fazem parte do Grupo de Estudo de Qualidade de Vida da OMS no Brasil ${ }^{6}$.

O WHOQOL-bref contém 26 questões, todas de natureza objetiva, sendo 2 gerais (qualidade de vida geral e satisfação com a saúde). As demais representam cada uma das 24 facetas que compõem o instrumento original, o WHOQOL-100. Os quatro domínios avaliados pela versão abreviada são: físico, psicológico, relações sociais e meio ambiente. As questões possuem quatro tipos de escalas de resposta: intensidade (nada-extremamente), capacidade (nada-completamente), avaliação (muito insatisfeito-muito satisfeito; muito ruim-muito bom) e frequência (nunca-sempre). Cada uma das alternativas corresponde a um valor numérico de 1 a $5^{6}$.

Essa pesquisa foi aprovada e protocolada pelo Comitê de Ética em Pesquisa da Universidade Federal da Paraíba sob protocolo de registro $n^{\circ} 600 / 2008$, sendo assegurados aos participantes os direitos de sigilo, voluntariado e desistência da participação da pesquisa.

\section{Análise estatística}

O tratamento dos dados foi feito utilizando-se o programa SPSS V.11.5. As 
variáveis sociodemográficas são demonstradas por meio da estatística descritiva. Já para a verificação do nível de correlação entre as variáveis sociodemográficas e de qualidade de vida foi utilizada a Correlação de Spearman. O nível de significância estatística adotado foi de 5\%.

\section{RESULTADOS}

Os resultados demonstraram que, dos 51 cuidadores avaliados, a maioria era do sexo feminino $(92,2 \% ; n=47)$, solteira $(43,1 \% ; n=22)$, mãe $(68,6 \% ; n=35)$ e havia frequentado a escola até o Ensino Fundamental incompleto (47\%; $\mathrm{n}=24)$, de acordo com a Tabela 1. Em relação à profissão, a de dona de casa foi apontada por $66,7 \% \quad(n=34)$, enquanto que $33,3 \%(n=17)$ afirmaram assumir papel em outras profissões, entre elas, auxiliar de enfermagem, agricultora e vendedora. A idade variou entre 19 e 63 anos, com média de 36,29ะ11,10 anos.

Em relação ao tempo de atuação como cuidador do paciente atual, houve variação de 1 a 24 anos (média de $6,96 \pm 6,41$ anos), enquanto o tempo de cuidado diário variou de 2 a 24 horas (média de 19,18 \pm 7,54 horas). No que se refere à saúde geral dos cuidadores, $68,6 \%(n=35)$ afirmaram não possuir nenhum tipo de problema de saúde, enquanto $31,4 \%(n=16)$ relataram apresentar algum agravo em sua saúde, entre os quais os mais citados foram diabetes, hipertensão arterial e algias na coluna vertebral.

O uso de medicamentos, consequente à presença de doença crônica, teve resultados proporcionais ao fato da maioria dos cuidadores não possuírem problemas de saúde. Dentre os cuidadores, 74,5\% $(n=38)$ não fazem o uso contínuo de medicamentos, enquanto que apenas $25,5 \%(n=13)$ utilizam algum tipo de fármaco.

Quando os cuidadores foram questionados se sentiam, ao final do dia, dor, incômodo ou desconforto no corpo, os resultados apontaram que $70,6 \%(n=36)$ relataram sentir algumas dessas sensações, enquanto $29,4 \%(n=15)$ afirmaram nada sentir ao final do dia. Dentre todas as regiões apontadas pelos cuidadores, $23,5 \%(n=12)$ afirmaram que a lombar da coluna vertebral é a que possui a

Tabela 1. Características sociodemográficas dos cuidadores

\begin{tabular}{|c|c|c|}
\hline Características sociodemográficas & $\mathrm{n}$ & $\%$ \\
\hline \multicolumn{3}{|l|}{ Sexo } \\
\hline Feminino & 47 & 92,2 \\
\hline Masculino & 4 & 7,8 \\
\hline \multicolumn{3}{|l|}{ Estado civil } \\
\hline Casado(a) & 19 & 37,3 \\
\hline Solteiro(a) & 22 & 43,1 \\
\hline Divorciado(a) & 1 & 2,0 \\
\hline Viúvo(a) & 2 & 3,9 \\
\hline Outros & 7 & 13,7 \\
\hline \multicolumn{3}{|l|}{ Grau de parentesco } \\
\hline Sem vínculo familiar & 5 & 9,9 \\
\hline Mãe & 35 & 68,6 \\
\hline Pai & 2 & 3,9 \\
\hline Avô(a) & 3 & 5,9 \\
\hline Irmão(a) & 2 & 3,9 \\
\hline Filho(a) & 2 & 3,9 \\
\hline Cônjuge & 2 & 3,9 \\
\hline \multicolumn{3}{|l|}{ Escolaridade } \\
\hline Sem escolaridade & 2 & 3,9 \\
\hline Ensino Fundamental incompleto & 24 & 47,0 \\
\hline Ensino Fundamental completo & 7 & 13,7 \\
\hline Ensino Médio incompleto & 3 & 5,9 \\
\hline Ensino Médio completo & 11 & 21,6 \\
\hline Ensino Superior incompleto & 3 & 5,9 \\
\hline Ensino Superior completo & 1 & 2,0 \\
\hline \multicolumn{3}{|l|}{ Profissão } \\
\hline Dona de casa & 34 & 66,7 \\
\hline Outras & 17 & 33,3 \\
\hline
\end{tabular}

n: valor absoluto

Fonte: Pesquisa Direta, 2008/2009

maior sintomatologia dolorosa no corpo; a região do pescoço ficou em segundo lugar, com $13,7 \%(n=7)$ e as demais regiões tiveram valores menores e/ou empatados.

Os aspectos da qualidade de vida dos cuidadores foram analisados pelos domínios do WHOQOL-bref e, neste estudo, os escores, do maior ao menor, respectivamente foram: relações sociais, índice geral de qualidade de vida (IGQV) e físico, com os três escores mais altos; enquanto domínio psicológico e meio ambiente posicionaram-se entre os mais baixos apontados pelos entrevistados (Tabela 2).

Quando as variáveis sociodemográficas dos cuidadores foram correlacionadas com os domínios da qualidade de vida, os resultados encontrados demonstraram que apenas a variável "escolaridade" apresentou valor de significância estatística, ou seja, $p<0,05$. Houve correlação direta ou positiva entre os itens escolaridade e domínio psicológico 0,275 ( $p=0,051)$.

Uma vez correlacionados os domínios de qualidade de vida e as variáveis sociodemográficas, buscou-se investigar se haveria também alguma relação entre as próprias variáveis sociodemográficas, ou seja, realizou-se um cruzamento entre todos os pontos presentes no perfil do cuidador. Na Tabela 3 observam-se os pontos que obtiveram correlação significativa. Os parâmetros que apresentaram correlação direta ou positiva entre si foram idade e tempo de atuação como cuidador do atual paciente $(p=0,000)$; e os de correlação inversa ou negativa foram escolaridade e tempo de cuidado diário com paciente $(p=0,009)$. 
Tabela 2. Resultado do World Health Organization Quality of Life segundo os domínios avaliados

\begin{tabular}{lc}
\hline Domínios do WHOQOL-bref & média \pm DP \\
\hline Índice geral de qualidade de vida & $61,02 \pm 16,32$ \\
Domínio físico & $59,38 \pm 10,07$ \\
Domínio psicológico & $57,35 \pm 11,59$ \\
Domínio relações sociais & $67,97 \pm 25,02$ \\
Domínio meio ambiente & $50,00 \pm 14,29$ \\
\hline
\end{tabular}

Fonte: Pesquisa Direta, 2008/2009

Tabela 3. Correlação entre as variáveis sociodemográficas dos cuidadores

\begin{tabular}{lcc}
\hline Variável & Idade & Escolaridade \\
\hline $\begin{array}{l}\text { Tempo de atuação como cuidador do } \\
\text { atual paciente }\end{array}$ & $0,513(0,000)^{*}$ & $-0,146(0,308)$ \\
$\begin{array}{l}\text { Tempo de cuidado diário com o } \\
\text { paciente }\end{array}$ & $0,027(0,850)$ & $-0,360(0,009)^{*}$ \\
\hline
\end{tabular}

*Valores significantes $(\mathrm{p}<0,05)$

Fonte: Pesquisa Direta, 2008/2009.

\section{DISCUSSÃO}

Os dados revelaram que grande porcentagem dos cuidadores de pessoas com deficiência física deste estudo eram mulheres jovens, solteiras, mães e com nível de escolaridade até o Ensino Fundamental incompleto, tendo sido observadas correlações entre estes perfis e o nível de qualidade de vida adotado por eles.

Quanto à saúde geral dos cuidadores, verificou-se que a maioria não fazia o uso de medicação. No entanto, em relação à presença ou não de dor, incômodo e/ou desconforto no corpo, ao final do dia a maior parte apontou sentir qualquer uma dessas sensações, principalmente na região lombar da coluna vertebral. Para Ferrari et al. ${ }^{8}$, essa sintomatologia dolorosa do cuidador esta relacionada à tensão emocional e ao esforço físico permanentes. O esforço contínuo é muitas vezes exigido no processo do cuidado, somado a outras responsabilidades do cuidador, como filhos, fatores esses que deterioram a sua saúde.

Em controvérsia com esses achados, uma pesquisa desenvolvida pela Universidade da Flórida (EUA), com 29.277 cuidadores de pessoas com deficiência, mapeou o estilo de vida e o nível de qualidade de saúde em 50 estados do país (incluindo o distrito da Carolina do Norte) e Porto Rico, utilizando o Behavioral Risk Factor Surveillance System, instrumento adotado pelo programa de saúde pública do governo americano, o Healthy People. Os resultados demonstraram que a maioria dos cuidadores era do sexo feminino, casados, formados $\left(2^{\circ}\right.$ grau completo), autônomos e, quando questionados sobre a sua própria saúde, $83,24 \%$ se referia como excelente ou muito boa. Em relação à idade, pode-se observar que aqueles com 55 anos ou menos tiveram o risco aumentado em $35 \%$ de ter uma saúde ruim ou muito ruim quando comparados aos que tinham mais de 55 anos, em que esse risco foi menor que $3 \%$. Dois aspectos corroboram-se entre a pesquisa norteamericana e este presente estudo: em ambos a maioria dos cuidadores eram mulheres com idade abaixo de 55 anos, ou seja, a mulher jovem, independente da escolaridade, é preferida para desempenhar o papel do cuidado, desenvolvendo essa atividade de forma remunerada ou não ${ }^{9}$.

Diferindo da perspectiva norteamericana, um estudo desenvolvido na Espanha ${ }^{10}$ com 153 cuidadores informais de pessoas idosas demonstrou que o perfil desta população era de mulheres $(62,7 \%)$ acima de 50 anos, com baixa escolaridade $(67,3 \%)$ e que não possuíam registro de emprego formal $(82,4 \%)$.
A idade avançada e a baixa escolaridade dos cuidadores estariam atreladas a sentimentos de baixa estima e falta de competência para continuar prestando o atendimento. Dados semelhantes foram encontrados numa pesquisa em Taiwan ${ }^{11}$, a qual envolvia 1.600 pessoas, entre cuidadores e pacientes, na qual a maioria eram mulheres $(67 \%)$, casadas ou que viviam com seus cônjuges $(79,8 \%)$, de média à alta escolaridade (86,9\%) e com boa percepção acerca da sua própria saúde $(83,5 \%)$.

Os resultados apresentados quanto à qualidade de vida dos cuidadores demonstraram que os domínios psicológico e meio ambiente foram os mais afetados. O baixo escore obtido no domínio psicológico é corroborado no estudo que avaliou a qualidade de vida de famílias de crianças com Síndrome de Down em Brasília (DF). Foi observado que o baixo escore do domínio psicológico na avaliação da qualidade de vida dos pais estaria relacionado a fatores como sobrecarga nos níveis social, psicológico e financeiro e também com relação aos cuidados com uma criança com deficiência física, que podem gerar sentimentos de ansiedade e incerteza. Devido a questões como segurança, nível socioeconômico e meio ambiente (poluição, ruído, trânsito e clima) afetarem a todos os cuidadores de maneira similar, independente de idade e atividade ${ }^{12}$, o item meio ambiente obteve o escore mais baixo neste presente estudo.

Em contrapartida, Lin et al. ${ }^{13}$ encontraram, em sua investigação sobre a qualidade de vida de 597 cuidadores de crianças com distúrbios intelectuais de Taiwan, que o domínio psicológico obteve o menor escore, justificando-se em razão dos anos de prestação de cuidados, o que gera certo grau de angústia no cuidador. Esse sentimento é associado às necessidades comportamentais e emocionais encontradas nas crianças com déficits intelectuais. Os baixos resultados verificados nos cuidadores, em todos os domínios do WHOQOL-bref, quando comparados à população geral elucidam a necessidade da implementação de políticas específicas, de início precoce e que busquem fornecer informações de fácil acesso a respeito dos cuidados com seu estilo de vida ${ }^{14,15}$. 
Corroborando com esses achados, um estudo com 27 cuidadores de pessoas com fibrose cística da Universidade da Carolina do Norte (EUA) apresentou os sintomas psicológicos que esses cuidadores desenvolviam ao longo dos anos de atendimento e o fato de estarem lidando com uma patologia terminal, ainda na idade jovem. Eles relataram que as angústias e frustrações vividas pelos pacientes diante das terapias medicamentosas e internações hospitalares causaram, mesmo após a morte dos pacientes, sensações de vazio, alívio, baixa crença sobre os medicamentos, etc ${ }^{16}$.

Algumas limitações deste estudo devem ser citadas. A amostra selecionada é pequena e a presente pesquisa é do tipo transversal. Logo, estudos prospectivos experimentais poderão verificar os efeitos e a evolução de uma determinada intervenção em grupo ou individual para estes cuidadores por certo período de tempo.
CONCLUSÃO

Os resultados deste estudo apontaram que cuidadores de pessoas com deficiência física desenvolvem, ao longo do tempo de cuidado, alterações físicas e mentais negativas na qualidade de sua saúde, sugerindo que essas modificações estariam sendo influenciadas, direta ou inversamente, por aspectos particulares do estilo de vida, principalmente pela idade, nível de escolaridade e tempo de atuação como cuidador.

\section{REFERÊNCIAS}

1. Brasil. Ministério da Saúde. Secretaria de Atenção à Saúde. Definição da pessoa com deficiência. Brasília: Ministério da Saúde, 1999.

2. IBGE - Instituto Brasileiro de Geografia e Estatística. Censo Demográfico de 2000.

3. Brasil. Ministério da Saúde. Secretaria de Atenção à Saúde. Secretaria de Gestão do Trabalho e da Educação na Saúde. Guia prático do cuidador. Brasília: Ministério da Saúde, 2008.

4. Floriani CA. Cuidador familiar: sobrecarga e proteção. Rev Bras Cancerol. 2004;50(4):341-5.

5. Alvarenga MRM, Amendola F, Oliveira MAC. Qualidade de vida dos cuidadores de pacientes dependentes no programa de saúde da família. Texto Contexto Enferm. 2008; 17(2):266-72.

6. Fleck MPA, Fachel O, Louzada S, Xavier M, Chachamovich E, Vieira G, et al. Desenvolvimento da versão em português do instrumento de avaliação de qualidade de vida da Organização Mundial da Saúde (WHOQOL-100). Rev Bras Psiquiatr. 1999;21(1):19-28.

7. Barba MC, Bergmann RS, Jotz GP, Paulo MG, Teixeira AR. Avaliação da qualidade de vida de cuidadores de idosos portadores de deficiência auditiva: influência do uso de próteses auditivas. Rev Arq Int Otorrinolaringol. 2008;12(3):479-89.

8. Ferrari RAM, Frigero M, Sousa AG, Zarameli RC. Avaliação da qualidade de vida de cuidadores de pacientes com sequelas neurológicas. ConScientiae Saúde. 2008;7(4):497-502.

9. Neugaard B, Andresen E, McKune SL, Jamoom E. Healthrelated quality of life in a national sample of caregivers: findings from the behavioral risk factor surveillance system. J Happiness Stud. 2008;9(4):559-75.

10. Garcés J, Carretero S, Ródenas F, Sanjosé V. Variables related to the informal caregivers' burden of dependent senior citizens in Spain. Arch Gerontol Geriatr. 2009;48(3):372-9.

11. Chang $H$. The correlation of home care with family caregiver burden and depressive mood: an examination of moderating functions. Int J Gerontol. 2009;3(3):170-80.

12. Dessen MA, Silva NLP. Famílias de crianças com síndrome de Down: sentimentos, modos de vida e estresse parental. Interação em Psicologia. 2006;10(2):183-94.

13. Lin JD, Hu J, Yen CF, Hsu SW, Lin LP, Loh CH, et al. Quality of life in caregivers of children and adolescents with intellectual disabilities: use of WHOQOL-BREF survey. Res Dev Disabil. 2009;30(6):1448-58.

14. Zaid T, Sudall C, Kauffmann L, Floranmi S, Khalil B, Morabito A. Physical outcome and quality of life after total esophagogastric dissociation in children with severe neurodisability and gastroesophageal reflux, from the caregiver's perspective. J Pediatr Surg. 2010;45(9):1772-6.

15. Gallop K, Wild D, Verdian L, Kerr M, Jacoby A, Baker $\mathrm{G}$, et al. Lennox-Gastaut Syndrome (LGS): development of conceptual models of health-related quality of life (HRQL) for caregivers and children. Seizure. 2010;19(1):23-30.

16. Dellon EP, Shores MD, Nelson KI, Wolfe J, Noah TL, Hanson LC. Family caregiver perspectives on symptoms and treatments for patients dying from complications of cystic fibrosis. J Pain Symptom Manage. 2010;40(6):829-37. 